\title{
VLBI astrometry of the semi-regular variable star RX Bootis
}

\author{
Tatsuya Kamezaki, ${ }^{1}$ Akiharu Nakagawa, ${ }^{1}$ Toshihiro Omodaka, ${ }^{1}$ \\ Toshihiro Handa, ${ }^{1}$ Hiroshi Imai, ${ }^{1}$ Daniel Tafoya, ${ }^{1}$ Makoto Matsui, ${ }^{1}$ \\ Yoshiro Nishida, ${ }^{1}$ Takumi Nagayama, ${ }^{2}$ Mareki Honma, ${ }^{2}$ \\ Hideyuki Kobayashi, ${ }^{2}$ Takeshi Miyaji, ${ }^{2}$ and Mine Takeuti ${ }^{3}$ \\ ${ }^{1}$ Department of Physics and Astronomy, Graduate School of Science and Engineering, \\ Kagoshima University, 1-21-35 Korimoto, Kagoshima, Kagoshima 890-0065, Japan \\ email: kamezaki@milkyway.sci.kagoshima-u.ac.jp \\ ${ }^{2}$ Mizusawa VLBI Observatory, Mitaka Office, National Astronomical Observatory of Japan, \\ 2-21-1 Osawa, Mitaka, Tokyo 181-8588, Japan \\ ${ }^{3}$ Astronomical Institute, Tohoku University, 6-3 Aramaki, Aoba-ku, Sendai 980-8578, Japan
}

\begin{abstract}
We present a distance measurement to the semi-regular variable star RX Bootis (RX Boo). Using the VLBI Exploration of Radio Astrometry (VERA) telescope, we conducted astrometric observations of a water maser spot associated with RX Boo, as well as of the continuum reference source J1419+2706. Based on monitoring observations covering a full year, the annual parallax of RX Boo was measured at $7.31 \pm 0.50$ mas, corresponding to a distance of $136_{-9}^{+10}$ pc. This distance uncertainty is smaller by a factor of two than those previously published, allowing us to determine the object's stellar properties more accurately. Using our distance, we can determine the absolute magnitude and discuss more precisely the locus of RX Boo on the period-luminosity (PL) relation. RX Boo exhibits two simultaneous pulsation periods and is located on the fundamental and first overtone Mira sequences of the PL relation. In addition, we calculated the radius and mass of the star.
\end{abstract}

Keywords. stars: AGB and post-AGB, stars: individual (RX Bootis), stars: late-type, astrometry

\section{Introduction}

Certain types of variable stars exhibit well-defined relations between their absolute magnitudes and their periods. Such relations are called period-luminosity (PL) relations. PL relations of long-period variable stars (for example, Miras or semi-regular - SRa or $\mathrm{SRb}$ - variable stars) have long been investigated, since they have been found in the Large Magellanic Cloud (Glass \& Evans 1981; Wood 2000; Cioni et al. 2001; Ita et al. 2004). By employing a PL relation, we can estimate a variable star's absolute magnitude based on its period. Armed with this absolute magnitude, and using the star's apparent magnitude from observations, we can calculate its distance. Detailed studies of PL relations have revealed an intrinsic multiplicity, a feature usually explained by differences among excited pulsation modes. For red, long-period variable stars, evidence of such multiplicity was first reported by Wood (2000).

Although PL relations in our Galaxy have been studied extensively (e.g., Knapp et al. 2003; Glass \& van Leeuwen 2007; Whitelock et al. 2008), further studies are required. One of the difficulties associated with the PL relations in our Galaxy is that of measuring precise distances. However, we must obtain precise distances to determine a precise PL relation. Therefore, we measured the distance to our sample object, RX Bootis (RX Boo), from its annual parallax using Very Long Baseline Interferometry (VLBI). 
We observed RX Boo with the Japanese VLBI telescope, 'VLBI Exploration of Radio Astrometry' (VERA). VERA consists of four antennas (located in Mizusawa, Iriki, Ogasawara, and Ishigakijima). It can measure the annual parallaxes and proper motions of many astronomical objects based on application of a phase-referencing method. Since VERA is a dual-beam system, we observed our target and reference sources simultaneously. Using the system, we can cancel out the effects of atmospheric fluctuations between two sources (Honma et al. 2008).

\section{Observations and Data Reduction}

We conducted monthly VLBI/VERA observations of water masers around RX Boo from February 2008 to October 2009. We performed a total of 12 epochs of observations. To obtain the positions of the maser spots of interest around RX Boo, we observed a continuum source $(\mathrm{J} 1419+2706)$ as an absolute position reference. The phase-tracking centers of RX Boo and J1419+2706 are $\left(\alpha_{\mathrm{J} 2000.0}, \delta_{\mathrm{J} 2000.0}\right)=\left(14^{\mathrm{h}} 24^{\mathrm{m}} 11^{\mathrm{s}} .6206,+25^{\circ} 42^{\prime}\right.$ $\left.12^{\prime \prime} .909\right)$ and $\left(\alpha_{\mathrm{J} 2000.0}, \delta_{\mathrm{J} 2000.0}\right)=\left(14^{\mathrm{h}} 19^{\mathrm{m}} 59^{\mathrm{s}} .2971,+27^{\circ} 06^{\prime} 25^{\prime \prime} .5530\right)$, respectively.

We reduced the data with the Astronomical Image Processing System (AIPS) developed by the National Radio Astronomical Observatory (NRAO). The data reduction method and our approach to estimate the uncertainties have been published in detail in a number of VERA papers (e.g., Honma et al. 2007; Nakagawa et al. 2008; Kamezaki et al. 2012).

\section{Results}

The spectral shapes did not change during the VLBI observations. Using least-squares fitting, we determined the annual parallax and proper motion based on the single maser spot we detected. We show the fit results in Figs 1 and 2. Based on these fits, we determined the parallax of RX Boo at $7.31 \pm 0.50$ mas, corresponding to a distance of $136_{-9}^{+10}$ $\mathrm{pc}$, as well as its proper motion, $\left(\mu_{\alpha} \cos \delta, \mu_{\delta}\right)=\left(24.55 \pm 1.06 \mathrm{mas} \mathrm{yr}^{-1},-49.67 \pm 2.38\right.$

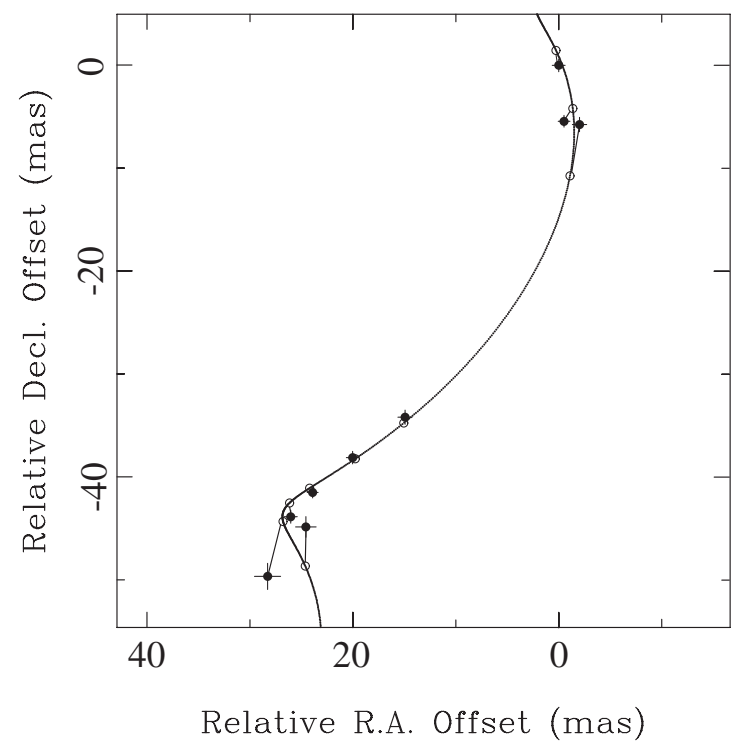

Figure 1. Relative position of the maser spot associated with RX Boo with respect to the phase-tracking center, $(\alpha, \delta)=\left(14^{\mathrm{h}} 24^{\mathrm{m}} 11^{\mathrm{s}} .6206,+25^{\circ} 42^{\prime} 12^{\prime \prime} .909\right)$. Solid bullets with error bars: observed positions; open circles: positions calculated from least-squares fitting. 


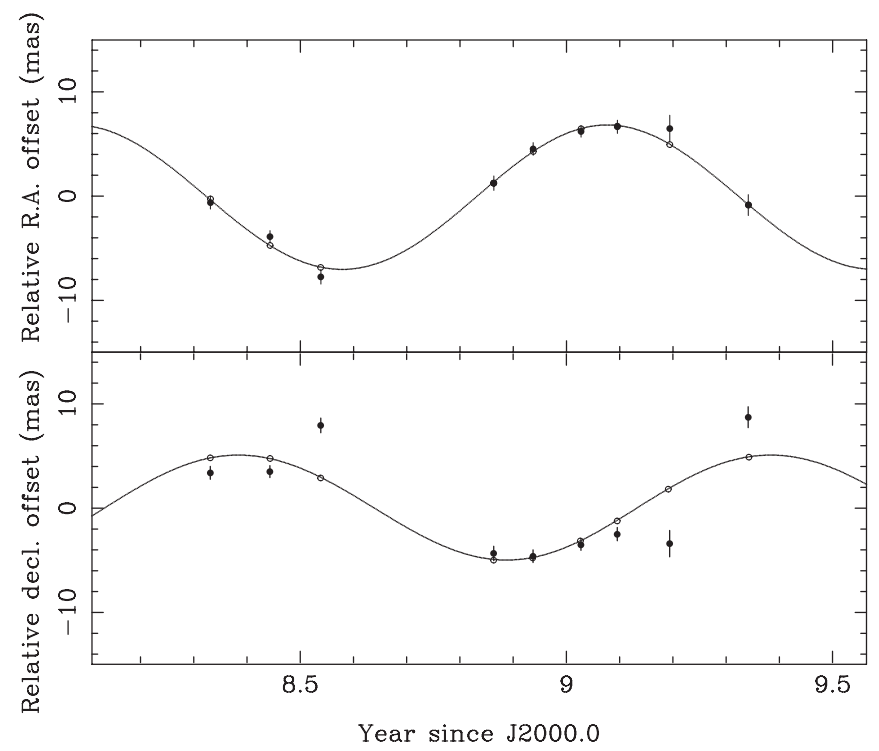

Figure 2. Time variation of the position of a maser spot near RX Boo in right ascension (top) and declination (bottom) after subtracting the linear proper motion. Solid line: fit result of the annual parallax; solid bullets with error bars: observed positions; open circles: positions calculated from least-squares fitting.

mas $\left.\mathrm{yr}^{-1}\right)$. The annual parallax measured by Hipparcos was $6.42 \pm 1.00$ mas; our parallax is consistent with the Hipparcos parallax. The Hipparcos proper motion was $\left(\mu_{\alpha} \cos \delta\right.$, $\left.\mu_{\delta}\right)=\left(21.74 \pm 0.90\right.$ mas $\mathrm{yr}^{-1},-49.70 \pm 0.49$ mas $\left.\mathrm{yr}^{-1}\right)$. Our proper motion and the Hipparcos proper motion are consistent in declination but differ in right ascension. This is because we observed a maser source near RX Boo, while Hipparcos observed the star itself. Therefore, the difference between our results and those based on Hipparcos data

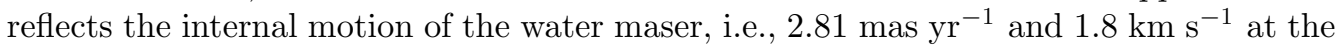
distance of RX Boo.

\section{Stellar Properties}

We will now discuss the position of RX Boo on the PL relation. The multiple periods of RX Boo have been studied previously (Taylor 1987; Andronov \& Kudashkina 1988; Mattei et al. 1997; Speil 2006; Samus et al. 2009). RX Boo exhibits two simultaneous periods, a shorter period of $\sim 160$ days and a longer one of $\sim 300$ days. We adopted 159.6 days and 278.0 days, respectively, based on the most recent study (Speil 2006). Using our distance, the absolute magnitude in the near-infrared $K$ band was estimated as $M_{K}=-7.53_{-0.15}^{+0.14} \mathrm{mag}$, based on an apparent magnitude of $m_{K}=-1.85 \mathrm{mag}$ (Glass $\&$ van Leeuwen 2007). The two periods were found on sequences $\mathrm{C}$ and $\mathrm{C}^{\prime}$, which describe the relation for Miras and SRa stars, and that for some SRb stars, respectively. RX Boo is found on sequence $\mathrm{C}$ if we consider the longer period, while it is located on sequence $\mathrm{C}^{\prime}$ for the short period. This indicates that both periods of RX Boo may be related to its basic stellar structural properties, and are unlikely due to any spontaneous occurrences. One of the periods corresponds to a definite mode of pulsation, and the other to another mode. The simultaneous enhancement of both modes may be evidence of the transient nature of RX Boo between sequence-C and $\mathrm{C}^{\prime}$ stars. 
We also estimated the radius of RX Boo, using two methods: one was based on our distance and the stellar diameter obtained from infrared interferometry, and the other used our distance and Stefan-Boltzmann's law. In application of the former method, we used the angular diameter of the Rosseland radius, $18.87 \pm 0.12$ mas, based on Perrin et al. (1998). The star's Rosseland radius of $278 \mathrm{R}_{\odot}$ was obtained by combining their radius and the present parallax. In the latter method, we estimated the bolometric luminosity using the $V$-band magnitude and a bolometric correction, and then we estimated the radius from Stefan-Boltzmann's law, the bolometric luminosity, and an effective temperature. The bolometric correction is $-6.5 \mathrm{mag}$ for $(V-K) \sim 9.85 \mathrm{mag}$ (Worthey \& Lee 2011). Since the mean $V$-band magnitude of RX Boo was $m_{V} \sim 8.0$ mag (e.g., AAVSO, AFOEV, GCVS, etc.), we estimated the apparent bolometric magnitude at $m_{\text {bol }} \sim 1.5$ mag. Finally, we obtained $M_{\mathrm{bol}}=-4.2 \mathrm{mag}$, which gives a stellar luminosity of $3630 \mathrm{~L}_{\odot}$. When we use an effective temperature of $2750 \mathrm{~K}$ based on Worthey \& Lee (2011), a radius of $266 \mathrm{R}_{\odot}$ is obtained. At present, we assume a radius for RX Boo of approximately $270 \mathrm{R}_{\odot}$ based on results obtained from two different procedures.

The radius of a pulsating star can be used to estimate the stellar mass when the characteristic period, the pulsation constant $(Q)$, is obtained from theoretical considerations. The detailed theoretical study of the radial pulsations of red giants performed by Xiong \& Deng (2007) gives the period ratio and $Q$ for various models. The period ratio of RX Boo is 0.61 for the results of Speil (2006) and we adopted $Q \sim 0.039$ from Xiong \& Deng (2007). From the period-density relation, we estimated the mass of RX Boo using the pulsation constant, radius, and period. We obtained a mass for RX Boo of $1.13 \mathrm{M}_{\odot}$. The mass estimated here will increase if we adopt a larger radius.

\section{References}

Andronov, I. L. \& Kudashkina, L. S. 1988, Astron. Nachr., 309, 323

Cioni, M.-R. L., Marquette, J.-B., Loup, C., Azzopardi, M., Habing, H. J., Lasserre, T., \& Lesquoy, E. 2001, A\& $A, 377,945$

Glass, I. S. \& Evans, T. L. 1981, Nature, 291, 303

Glass, I. S. \& van Leeuwen, F. 2007, MNRAS, 378, 1543

Honma, M., Bushimata, T., Choi, Y. K., et al. 2007, PASJ, 59, 889

Honma, M., Kijima, M., Suda, H., et al. 2008, PASJ, 60, 935

Ita, Y., Tanabe, T., Matsunaga, N., et al. 2004, MNRAS, 353, 705

Kamezaki, T., Nakagawa, A., Omodaka, T., et al. 2012, PASJ, 64, 7

Knapp, G. R., Pourbaix, D., Platais, I., \& Jorissen, A. 2003, A\&SA, 403, 993

Mattei, J. A., Foster, G., Hurwitz, L. A., Malatesta, K. H., Willson, L. A., \& Mennessier, M. O. 1997, in: Proc. ESA Symp. "Hipparcos - Venice '97" (Bonnet, R.M., Høg, E., Bernacca, P.L., et al., eds.), ESA-SP, 402, 269

Nakagawa, A., Tsushima, M., Ando, K., et al. 2008, PASJ, 60, 1013

Perrin, G., Coudé du Foresto, V., Ridgway, S. T., Mariotti, J.-M., Traub, W. A., Carleton, N. P., \& Lacasse, M. G. 1998, A\&A, 331, 619

Samus, N. N., Durlevich, O. V., et al. 2009, General Catalogue of Variable Stars (VizieR Online Data Catalog), Inst. of Astron., Russ. Acad. Sci. \& Sternberg, State Astron. Inst., Moscow State Univ.

Speil, J. 2006, JAAVSO, 35, 88

Taylor, M. D. 1987, J. Brit. Astron. Assoc., 97, 277

Worthey, G. \& Lee, H.-C. 2011, ApJS, 193, 1

Wood, P. R. 2000, Publ. Astron. Soc. Aus., 17, 18

Whitelock, P. A., Feast, M. W., \& van Leeuwen, F. 2008, MNRAS, 386, 313

Xiong, D. R. \& Deng, L. 2007, MNRAS, 378, 1270 